\title{
DIMENSION OF SUBSETS OF PRODUCT SPACES
}

\author{
Y. STERNFELD
}

\begin{abstract}
It is proved that under a certain condition on a separable metric space $X$, each compact subset $W$ of $X \times Y$ with $\operatorname{dim} W=\operatorname{dim} X+\operatorname{dim} Y$ contains a product $X^{\prime} \times Y^{\prime} \subset W$ with $\operatorname{dim} X^{\prime}=\operatorname{dim} X$ and $\operatorname{dim} Y^{\prime}=\operatorname{dim} Y$. This condition is satisfied when $X$ is a Euclidean space.
\end{abstract}

A classical result of dimension theory states that a subset of the Euclidean $n$ space $\mathbf{R}^{n}$ has dimension $n$ if and only if it has a nonempty interior in $\mathbf{R}^{n}$. A different way to state the same result is the following: For $W \subset \mathbf{R}^{n}, \operatorname{dim} W=n$ if and only if $W$ contains an $n$-dimensional rectangle, i.e., a product $\prod_{i=1}^{n} I_{i}$ where $I_{i}$ is a nondegenerate interval, $1<i<n$. In this note we wish to extend this result (in its second statement) to product spaces other than $\mathbf{R}^{n}$, and study the following question:

Let $X$ and $Y$ be finite-dimensional separable metric spaces, and let $W$ be a subset of $X \times Y$ with $\operatorname{dim} W=\operatorname{dim} X+\operatorname{dim} Y$. Under which conditions will it follow that $W$ contains a "rectangle" of the form $X^{\prime} \times Y^{\prime}$ with $\operatorname{dim} X^{\prime}=\operatorname{dim} X$ and $\operatorname{dim} Y^{\prime}=\operatorname{dim} Y$.

We shall see that compactness of $W$ and a certain countability condition on one of the factors $X$ or $Y$ (which is satisfied when $X$ (or $Y$ ) is Euclidean) imply a positive answer to our question, while without compactness of $W$ it fails even in the simplest possible case, namely when $X$ is an interval and $Y$ the Cantor set. We do not know what the answer to this problem is if we just assume compactness of $W$.

To state our main result we introduce the following terminology:

DEFINITION. Let $X$ be a separable metric space.

1. A family $Q$ of closed subsets of $X$ is said to be a dimensional network for $X$ if $\operatorname{dim} H=\operatorname{dim} X$ for all $H \in \mathbb{Q}$, and if each closed $F \subset X$ with $\operatorname{dim} F=\operatorname{dim} X$ contains some element of $\mathcal{Q}$.

2. $X$ is said to be countably $n$-dimensional if $\operatorname{dim} X=n$ and it has a countable dimensional network.

Note that a space is countably zero dimensional if and only if it countable, that the space $\mathbf{R}^{n}$ is countably $\boldsymbol{n}$-dimensional, and that for any $\boldsymbol{n}$-dimensional space $X, X \times \Delta$ is an $n$-dimensional space which fails to be countably $n$-dimensional, where $\Delta$ denotes the Cantor set.

It turns out that the above question has a positive answer if $W$ is compact and one of the factors $X$ or $Y$ has its dimension countably.

Received by the editors February 20, 1980 and, in revised form, October 29, 1980.

AMS (MOS) subject classifications (1970). Primary 54F45. 
Theorem 1. Let $X$ and $Y$ be separable metric spaces with $X$ countably $(\operatorname{dim} X)$ dimensional, and let $W \subset X \times Y$ be compact with $\operatorname{dim} W=\operatorname{dim} X+\operatorname{dim} Y$. Then there exist compact sets $X^{\prime} \subset X, Y^{\prime} \subset Y$, with $\operatorname{dim} X^{\prime}=\operatorname{dim} X, \operatorname{dim} Y^{\prime}=\operatorname{dim} Y$ and $X^{\prime} \times Y^{\prime} \subset W$.

The compactness of $W$ in Theorem 1 cannot be omitted. It follows from the theorem that it will still hold if we assume that $W$ is $\sigma$-compact. But it turns out that Theorem 1 fails if we assume that $W$ is an aboslute $G_{8}$. Indeed, in [3, pp. 80-81], following Knaster, Lelek constructs an $n$-dimensional totally disconnected $G_{\delta}$ subset of $\mathbf{R}^{n+1}$. It easily follows from the construction that this set is homeomorphic to a subset $W$ of $I^{n} \times \Delta$, such that for each $t \in \Delta,\left(I^{n} \times\{t\}\right) \cap W$ consists of a single point. In particular $W$ does not contain any set of the form $X \times Y$, $X \subset I^{n}, Y \subset \Delta$, for which $X$ contains more than one point. (Here $I=[-1,1]$ and $\Delta$ is the Cantor set.)

As mentioned above we do not know whether the countability condition in Theorem 1 is really needed; we state this as a problem:

Problem 1. Let $W$ be a compact subset of $X \times Y$ with $\operatorname{dim} W=\operatorname{dim} X+$ $\operatorname{dim} Y(X, Y$ separable metric spaces). Does it follow that $W$ contains a product $X^{\prime} \times Y^{\prime}$ with $\operatorname{dim} X^{\prime}=\operatorname{dim} X$ and $\operatorname{dim} Y^{\prime}=\operatorname{dim} Y ?$ In particular does the above hold if $\operatorname{dim} X=\operatorname{dim} Y=1$ ? (If $\operatorname{dim} X$ or $\operatorname{dim} Y=0$ the answer is positive.)

For the proof of Theorem 1 we shall need the following result of Jung and Keesling [1], [2].

The Jung-Keesling Theorem. Let $P$ be a closed mapping of an $(n+k)$ dimensional metric space $W$ onto a $k$-dimensional space $Y$. Set $D=\{y \in Y$ : $\left.\operatorname{dim} P^{-1}(y)>n\right\}$. Then $\operatorname{dim} W=n+k<\operatorname{dim} D+\operatorname{dim} P$, where $\operatorname{dim} P=$ $\sup _{y \in Y} \operatorname{dim} P^{-1}(y)$.

Proof of Theorem 1. Set $\operatorname{dim} X=n, \operatorname{dim} Y=k$. Let $P$ denote the restriction to $W$ of the natural projection of $X \times Y$ onto $Y$. For $y \in Y, P^{-1}(y)$ is homeomorphic to a subset of $X$, hence $\operatorname{dim} P^{-1}(y)<n$, i.e., $\operatorname{dim} P<n$. The compactness of $W$ implies that $P$ is a closed mapping, and by the Jung-Keesling Theorem, $\operatorname{dim} D>(n+k)-n=k$, where $D$ is the set defined in this theorem. For a closed subset $F$ of $X$ set $\varphi(F)=\{y \in Y: F \times\{y\} \subset W\} . \varphi(F)$ is closed in $Y$, since by its definition $F \times \varphi(F) \subset W$, and by the compactness of $W$, $F \times \overline{\varphi(F)} \subset \overline{F \times \varphi(F)} \subset W$.

Let $\mathcal{Q}$ be a countable dimensional network for $X$. We claim that $D \subset$ $\cup_{F \in \mathbb{Q}} \varphi(F)$. Indeed, let $y \in D$. Then $\operatorname{dim} P^{-1}(y)=n$, and by the definition of a dimensional network there exists some $F \in \mathbb{Q}$ such that $P^{-1}(y)=(X \times\{y\}) \cap W$ $\supset F \times\{y\}$. In particular, $F \times\{y\} \subset W$ and $y \in \varphi(F)$.

It follows that $\operatorname{dim} \cup_{F \in \mathbb{Q}} \varphi(F)>\operatorname{dim} D>k$, and since the sets $\varphi(F)$ are closed, $\operatorname{dim} \varphi(F)>k$ for some $F \in \mathbb{Q}$. We also have $\operatorname{dim} F=n$ and $F \times \varphi(F) \subset W$ and the theorem follows.

Finally, let us state two more problems both related to a possible inequality in the product theorem. 
Problem 2. Can one have $\operatorname{dim}\left(X^{\prime} \times Y^{\prime}\right)=\operatorname{dim} W$ in Theorem 1 and in Problem $1 ?$

Problem 3. What can be said if the condition $\operatorname{dim} W=\operatorname{dim} X+\operatorname{dim} Y$ is replaced by $\operatorname{dim} W=\operatorname{dim}(X \times Y)$ in Theorem 1 and Problem 1 ?

\section{REFERENCES}

1. C. F. K. Jung, Mappings on compact metric spaces, Colloq. Math. 19 (1968), 73-76.

2. J. Keesling, Closed mappings which lower dimension, Colloq. Math. 20 (1969), 237-241.

3. A. Lelek, Dimension inequalities for unions and mappings of separable metric spaces, Colloq. Math. 23 (1971), 69-91.

Department of Mathematics, University of Haifa, HaIfa, IsRael 